\title{
Pulmonary large cell neuroendocrine carcinoma with adenocarcinoma-like features: napsin A expression and genomic alterations
}

\author{
Natasha Rekhtman ${ }^{1}$, Catherine M Pietanza ${ }^{2,5}$, Joshua Sabari ${ }^{2}$, Joseph Montecalvo ${ }^{1}$, \\ Hangjun Wang ${ }^{1,6}$, Omar Habeeb ${ }^{1,7}$, Kyuichi Kadota ${ }^{1,8}$, Prasad Adusumilli ${ }^{3}$, \\ Charles M Rudin ${ }^{2}$, Marc Ladanyi ${ }^{1,4}$, William D Travis ${ }^{1}$ and Philippe Joubert ${ }^{1,9}$
}

${ }^{1}$ Department of Pathology, Memorial Sloan Kettering Cancer Center, New York, NY, USA; ${ }^{2}$ Thoracic Oncology Service, Department of Medicine, Division of Solid Tumor Oncology, Memorial Sloan Kettering Cancer Center, New York, NY, USA; ${ }^{3}$ Department of Thoracic Surgery, Memorial Sloan Kettering Cancer Center, New York, NY, USA and ${ }^{4}$ Human Oncology and Pathogenesis Program, Memorial Sloan Kettering Cancer Center,

New York, NY, USA

Pulmonary large cell neuroendocrine carcinoma (LCNEC) is a highly aggressive malignancy, which was recently found to comprise three major genomic subsets: small cell carcinoma-like, non-small cell carcinoma (predominantly adenocarcinoma)-like, and carcinoid-like. To further characterize adenocarcinoma-like subset, here we analyzed the expression of exocrine marker napsin A, along with TTF-1, in a large series of LCNECs $(n=112)$, and performed detailed clinicopathologic and genomic analysis of napsin A-positive cases. For comparison, we analyzed napsin A expression in other lung neuroendocrine neoplasms (177 carcinoids, 37 small cell carcinomas) and 60 lung adenocarcinomas. We found that napsin A was expressed in $15 \%$ of LCNEC (17/112), whereas all carcinoids and small cell carcinomas were consistently negative. Napsin A reactivity in LCNEC was focal in 12/17 cases, and weak or moderate in intensity in all cases, which was significantly lower in the extent and intensity than seen in adenocarcinomas $(P<0.0001)$. The combination of TTF-1-diffuse/napsin A-negative or focal was typical of LCNEC but was rare in adenocarcinoma, and could thus serve as a helpful diagnostic clue. The diagnosis of napsin A-positive LCNECs was confirmed by classic morphology, diffuse labeling for at least one neuroendocrine marker, most consistently synaptophysin, and the lack of distinct adenocarcinoma component. Genomic analysis of 14 napsin A-positive LCNECs revealed the presence of mutations typical of lung adenocarcinoma (KRAS and/or STK11) in 11 cases. In conclusion, LCNECs are unique among lung neuroendocrine neoplasms in that some of these tumors exhibit low-level expression of exocrine marker napsin A, and harbor genomic alterations typical of adenocarcinoma. Despite the apparent close biological relationship, designation of adeno-like LCNEC as a separate entity from adenocarcinoma is supported by their distinctive morphology, typically diffuse expression of neuroendocrine marker(s) and aggressive behavior. Further studies are warranted to assess the clinical utility and optimal method of identifying adenocarcinoma-like and other subsets of LCNEC in routine practice.

Modern Pathology (2018) 31, 111-121; doi:10.1038/modpathol.2017.110; published online 8 September 2017

Correspondence: Dr N Rekhtman, MD, PhD, Department of Pathology, Memorial Sloan Kettering Cancer Center, 1275 York Avenue, New York, NY 10065, USA.

E-mail: rekhtman@mskcc.org

${ }^{5}$ Current address: Merck Research Laboratories, Rahway, NJ, USA ${ }^{6}$ Current address: Department of Pathology, McGill University, Jewish General Hospital, Montreal, QC, Canada

${ }^{7}$ Current address: Wellington Hospital, Wellington, New Zealand ${ }^{8}$ Current address: Department of Diagnostic Pathology, Kagawa University, Kagawa, Japan

${ }^{9}$ Current address: Department of Pathology, Quebec Heart and Lung Institute, Quebec, QC, Canada

Received 1 April 2017; revised 19 July 2017; accepted 20 July 2017; published online 8 September 2017
Pulmonary large cell neuroendocrine carcinoma (LCNEC) is a highly aggressive malignancy, whose biological relationship with small cell versus nonsmall cell carcinoma has been a matter of a longstanding debate. ${ }^{1}$ These tumors are defined by a constellation of features, which include (1) neuroendocrine morphology (nesting, peripheral palisading, and rosettes), (2) expression of neuroendocrine markers (synaptophysin, chromogranin A, and CD56), and (3) proliferation rate of $>10$ mitoses/10 high-power fields $\left(2 \mathrm{~mm}^{2}\right)$, although in the vast majority of cases, proliferation rates far exceed this 
threshold. ${ }^{2}$ These tumors are distinguished from small cell carcinoma by cytologic features, including prominent nucleoli and/or more abundant cytoplasm, and typically larger nuclear size. However, existence of a spectrum of morphology in tumors classified as LCNEC is well known, with some cases exhibiting overt non-small cell features, while others closely approaching the morphology of small cell carcinoma. ${ }^{3-5}$ Recently, using next-generation sequencing, we identified that LCNECs are molecularly heterogeneous, comprising three major molecular subsets: small cell-like (characterized by the joint RB1/TP53 inactivation), non-small cell/ adenocarcinoma-like (characterized by the lack of RB1/TP53 co-alterations and frequent KRAS and/or STK11 mutations), and in a minority of casescarcinoid-like (characterized by MEN1 mutations and low total mutation burden). ${ }^{6}$ Notably, these molecular subsets could be predicted morphologically in most but not all cases. ${ }^{6}$ Recently, small cell and adeno-like subsets of LCNEC were also identified in an expression profiling study. ${ }^{7}$ In both studies, it was noted that some adeno-like tumors exhibited expression of a relatively recent pulmonary exocrine marker-napsin A. However, this finding was not explored in detail.

Napsin A (Novel aspartic proteinase of the pepsin family) is an enzyme involved in surfactant protein maturation, which is expressed in type II pneumocytes and in the majority of lung adenocarcinomas. ${ }^{8}$ In the recent years, napsin $\mathrm{A}$ has emerged as a useful and broadly utilized diagnostic marker, which is helpful for confirming lung origin in carcinomas of unknown primary and for distinguishing lung adenocarcinomas from other pulmonary neoplasms, such as squamous cell carcinoma and mesothelioma. ${ }^{9-12}$ Prior to napsin A, the predominant marker of lung glandular differentiation has been TTF-1. However, TTF-1 is not only a marker of pneumocytes lineage, but it is also a putative regulator of neurogenesis, which is expressed in small cell carcinoma and LCNEC of various sites. ${ }^{13-15}$ This dual role of TTF-1 precludes its' use as a marker of exocrine differentiation in lung tumors. Thus, emergence of napsin A presents a new opportunity to assess exocrine differentiation in LCNEC.

The literature on napsin A expression in LCNEC and other lung neuroendocrine neoplasms is relatively limited. Few available studies to date have focused primarily on napsin A expression in carcinoid tumors and small cell carcinomas, finding those tumors to be consistently napsin A-negative. ${ }^{12,16,17}$ To our knowledge, only two prior studies included large series of LCNECs in the analysis of napsin A expression, and reported consistent absence of napsin $\mathrm{A}$ in those tumors. ${ }^{16,18}$ This contrasts with the finding from the above two studies showing napsin A expression in some LCNECs with adenolike molecular features. ${ }^{6,14,19}$ In addition, in our clinical practice, we have encountered a number of cases with classical features of LCNEC, which showed convincing labeling for napsin A. The goal of this study was therefore to clarify these conflicting observations regarding napsin A expression in LCNECs. Here we report on the analysis of napsin A along with TTF-1 expression in 112 LCNEC with detailed clinicopathologic and molecular characterization of napsin A-positive cases. For comparison, we analyzed napsin A expression in 177 carcinoids, 37 small cell carcinomas, and 60 lung adenocarcinomas.

\section{Materials and methods}

\section{Samples}

The study was performed with the approval of the Memorial Sloan Kettering Cancer Center (New York, NY) Institutional Review Board. Napsin A expression was analyzed in a total of 112 LCNECs, of which 69 cases were represented in tissue microarrays and 43 cases in whole-tissue sections. The latter group of tumors was also included in our prior publication. ${ }^{6}$ All cases with napsin A expression identified in tissue microarrays were subsequently analyzed using whole-tissue sections. Other analyzed lung neuroendocrine tumors included typical carcinoids $(n=88)$, atypical carcinoids $(n=89)$, and small cell carcinomas $(n=37)$, which were studied in tissue microarrays. Tissue microarrays were constructed from surgical resection specimens performed at our institution between 1992 and 2007. The tumors were sampled in triplicate using 2-mm cores from representative tumor areas. As a control group, 60 consecutively resected lung adenocarcinomas were analyzed in whole-tissue sections.

\section{Immunohistochemistry}

Immunohistochemistry was performed on a Ventana Discovery XT-automated stainer (Ventana Medical Systems, Tucson, AZ, USA). Tissue microarrays were analyzed using napsin A polyclonal antibody (rabbit polyclonal antibody; Ventana Medical Systems, Tucson, AZ, USA) and TTF-1 (mouse monoclonal; 8G7G3/1; Dako, Carpinteria, CA, USA). Given the data on potential lack of specificity of polyclonal napsin A antibody, ${ }^{11,20}$ all positive cases detected in tissue microarrays with polyclonal antibody were retested in whole-tissue section using both polyclonal and monoclonal napsin A antibodies (mouse monoclonal; clone IP64; Leica Biosystems, Buffalo Grove, IL, USA). Percentage of napsin A-immunoreactive tumor cells and the intensity of staining $(1+=$ weak; $2+=$ moderate; $3+=$ strong $)$ was recorded. Whole-tissue sections from all napsin A-positive cases were additionally studied by immunohistochemistry with the following primary antibodies: synaptophysin (mouse monoclonal, clone Snp88; BioGenex, Fremont, CA, USA), chromogranin A (mouse monoclonal; clone LK2H10; 
Ventana Medical Systems, Tucson, AZ, USA), CD56 (mouse monoclonal; clone MRQ-42; Ventana Medical Systems, Tucson, AZ, USA), TTF-1 (see above), and Ki67 (mouse monoclonal; clone MIB1; Dako, Carpinteria, CA, USA).

\section{Molecular Studies}

The cases were analyzed using methods that were previously described in detail elsewhere. These included Sequenom mass spectrometry genotyping for 92 hot-spot mutations in 8 oncogenes (including EGFR and KRAS), ${ }^{21}$ fragment analysis for EGFR exon 19 and ERBB2 (HER2) deletions, ${ }^{21}$ and Illumina-based next-generation sequencing using MSK-IMPACT (Memorial Sloan Kettering-Integrated Mutation Profiling of Actionable Cancer Targets) platform for 341 (v3) or 410 (v4) cancer genes. ${ }^{22}$

\section{Statistical Analysis}

Statistical analysis was carried out using GraphPad software (GraphPad Software Inc, La Jolla, CA, USA). $P$ values were calculated using Fisher's exact or Student's $t$-test.

\section{Results}

As summarized in Table 1, analysis of 326 neuroendocrine neoplasms revealed that napsin A labeling was absent in all typical carcinoids $(n=88)$, atypical carcinoids $(n=89)$, and small cell carcinomas $(n=37)$. In contrast, napsin A reactivity was detected in a total of 17 of 112 (15\%) LCNECs, which included 8/69 $(12 \%)$ cases analyzed in tissue microarrays, and 9/43 $(21 \%)$ of cases tested in whole-tissue sections.

All napsin A-positive LCNECs $(n=17)$ identified in the above screen were further studied in wholetissue sections. First, napsin A expression detected was confirmed in whole-tissue sections using both polyclonal and monoclonal napsin A antibodies, which showed identical reactivities. Second, as shown in Table 2, quantitation of napsin A expression showed that labeling was focal $(\leq 50 \%$ of tumor

Table 1 Screen of napsin A expression in lung neuroendocrine neoplasms

\begin{tabular}{lcc}
\hline & $\begin{array}{c}\text { Number } \\
\text { tested }\end{array}$ & $\begin{array}{c}\text { Number (\%) } \\
\text { napsin A-positive }\end{array}$ \\
\hline Typical carcinoid (TMA) & 88 & 0 \\
Atypical carcinoid (TMA) & 89 & 0 \\
Small cell carcinoma (TMA) & 37 & 0 \\
\multicolumn{2}{l}{ Large cell neuroendocrine carcinoma } & \\
All cases combined & 112 & $17(15 \%)$ \\
TMA & 69 & $8(12 \%)$ \\
Whole-tissue sections & 43 & $9(21 \%)$ \\
\hline
\end{tabular}

Abbreviation: TMA, tissue microarrays. cells labeling) in $71 \%(12 / 17)$ of cases, while in the remaining five cases $(20 \%)$ expression was diffuse (70-90\% of tumor cells labeling). In all cases, the intensity of napsin A labeling was weak to moderate $(1-2+)$, with none of the cases showing $(3+)$ intensity. The overall mean \pm s.d. for the percentage of positive tumor cells and intensity of labeling for napsin A was $34 \pm 32 \%$ and $1.4 \pm 0.4 \%$, respectively. This extent and intensity of napsin A labeling was significantly lower than that seen in lung adenocarcinomas, which typically showed more diffuse staining for napsin A (93 $\pm 34 \%$; $P=<0.0001)$ with significantly higher intensity $(2.6 \pm 1.4 ; P=<0.0001)$ (Table 3). In particular, in contrast to LCNECs, the vast majority of adenocarcinomas showed strong (3+) intensity of napsin A labeling. Representative cases of napsin A-positive LCNECs are illustrated in Figure 1 and Figure 2, and contrast in the extent and intensity of labeling with adenocarcinomas is illustrated in Figure 3. Notably, given that intense napsin A reactivity in entrapped pneumocytes and alveolar histiocytes is a wellknown pitfall for false-positive interpretation of napsin $\mathrm{A}$ as tumor cell staining, ${ }^{23}$ we ensured that in all cases, positive labeling was clearly present in tumor cells (see Figures 1 and 2).

Analysis of joint expression of napsin A and TTF-1 in LCNEC versus lung adenocarcinomas (Table 3) showed that the most common profile in LCNEC was TTF-1-positive/napsin A-negative (44\%), whereas this dichotomous profile was rare in adenocarcinomas $(3 \% ; 2 / 60$ cases). In both adenocarcinomas with TTF-1 expression in the absence of napsin A, TTF-1 was only weak/focal, whereas in LCNEC, TTF-1 expression was typically strong/diffuse in the absence of napsin A.

The review of morphology of napsin A-positive LCNECs in whole-tissue sections confirmed the classic features of LCNEC. Key features included organoid architecture, with tumor cells organized into solid nests and/or trabeculae with peripheral palisading, and rosette-like structures in most cases. Cytologically, tumor cells displayed prominent nucleoli and/or moderate to abundant cytoplasm. All cases showed high Ki67 proliferation index (mean, 60\%; range, 40-80\%) (Table 2). Despite the high proliferation rate, the tumors tended to have relatively uniform/monotonous round nuclei, typical of neuroendocrine neoplasms. There was no evidence of distinct conventional adenocarcinoma morphology in the form of lepidic, acinar, papillary, or micropapillary structures.

All napsin A-positive LCNECs were confirmed to label for at least one neuroendocrine marker in $\geq 10 \%$ of tumor cells (Table 2). Synaptophysin was positive in all 17 cases, with diffuse labeling ( $>50 \%$ tumor cells staining) in 14/17 (82\%) cases. Staining for chromogranin A and CD56 was more variable, with reactivity in $65 \%$ and $67 \%$ of cases, respectively. In cases where staining for napsin A and neuroendocrine markers was available on 
Table 2 Analysis of napsin A-positive large cell neuroendocrine carcinomas in whole-tissue sections

\begin{tabular}{|c|c|c|c|c|c|c|c|}
\hline & \multicolumn{2}{|c|}{ Napsin A } & \multirow{2}{*}{$T T F-1$} & \multirow{2}{*}{ Synapto-physin } & \multirow{2}{*}{ Chromogranin } & \multirow{2}{*}{ CD56 } & \multirow{2}{*}{ Ki67 } \\
\hline & Percentage & Intensity & & & & & \\
\hline Case 1 & $10 \%$ & $1+$ & Dif & Dif & Foc & 0 & $40 \%$ \\
\hline Case 2 & $50 \%$ & $1.5+$ & Dif & Dif & 0 & 0 & $50 \%$ \\
\hline Case 3 & $5 \%$ & $1.5+$ & Dif & Dif & Dif & Dif & $50 \%$ \\
\hline Case 4 & $70 \%$ & $1.5+$ & Dif & Foc & 0 & Dif & $80 \%$ \\
\hline Case 5 & $10 \%$ & $1+$ & Dif & Dif & Foc & 0 & $50 \%$ \\
\hline Case 6 & $10 \%$ & $1+$ & Dif & Dif & Dif & Foc & $50 \%$ \\
\hline Case 7 & $10 \%$ & $1.5+$ & Dif & Foc & Foc & Dif & $60 \%$ \\
\hline Case 8 & $5 \%$ & $1+$ & Foc & Dif & Dif & 0 & $50 \%$ \\
\hline Case $9(\text { ID } 42)^{\mathrm{a}}$ & $10 \%$ & $2+$ & NA & Dif & 0 & NA & $50 \%$ \\
\hline Case $10(\text { ID } 49)^{\mathrm{a}}$ & $90 \%$ & $2+$ & Dif & Dif & Dif & NA & $40 \%$ \\
\hline Case 11 (ID 28) $^{\mathrm{a}}$ & $20 \%$ & $1+$ & Dif & Dif & 0 & Dif & $80 \%$ \\
\hline Case $12(\text { ID } 32)^{\mathrm{a}}$ & $80 \%$ & $2+$ & Dif & Dif & 0 & Dif & $80 \%$ \\
\hline Case 13 (ID 59) $^{\mathrm{a}}$ & $90 \%$ & $2+$ & Dif & Dif & Dif & 0 & $60 \%$ \\
\hline Case 14 (ID 38) & $70 \%$ & $1.5+$ & Dif & Dif & 0 & Dif & $70 \%$ \\
\hline Case 15 (ID 40) $^{\mathrm{a}}$ & $20 \%$ & $1.5+$ & Dif & Dif & Foc & Foc & $80 \%$ \\
\hline Case 16 (ID 39) $^{\mathrm{a}}$ & $10 \%$ & $1+$ & Dif & Dif & Dif & Dif & $70 \%$ \\
\hline Case $17($ ID 64) & $20 \%$ & $1+$ & Dif & Foc & Foc & Foc & $50 \%$ \\
\hline Summary & $\begin{array}{c}\text { Mean } \pm \text { s.d.: } \\
34 \pm 32 \%\end{array}$ & $\begin{array}{c}\text { Mean } \pm \text { s.d.: } \\
1.4 \pm 0.4\end{array}$ & $\begin{array}{l}\% \text { positive: } \\
100 \%\end{array}$ & $\begin{array}{l}\% \text { positive: } \\
100 \%\end{array}$ & $\begin{array}{c}\% \text { positive: } \\
65 \%\end{array}$ & $\begin{array}{c}\% \text { positive: } \\
67 \%\end{array}$ & $\begin{array}{c}\text { Mean } \pm \text { s.d.: } \\
60 \pm 14 \%\end{array}$ \\
\hline
\end{tabular}

Abbreviations: Foc, focal ( $\leq 50 \%$ tumor cells labeling); Dif, diffuse ( $>50 \%$ tumor cells labeling); NA, not available.

${ }^{a}$ Shown in parentheses are corresponding case IDs from Rekhtman et al. ${ }^{6}$

Table 3 Comparison of napsin A and TTF-1 expression in large cell neuroendocrine carcinoma versus lung adenocarcinoma

\begin{tabular}{|c|c|c|c|}
\hline & $\operatorname{LCNEC}(\mathrm{n}=112)$ & Adenocarcinoma $(\mathrm{n}=60)$ & $\mathrm{P}$ value \\
\hline \multicolumn{4}{|l|}{ Napsin A expression } \\
\hline Napsin A-positive: $n(\%)$ & $17(15 \%)$ & $51(85 \%)$ & 0.0001 \\
\hline Extent of labeling in positive cases: mean \pm s.d. & $34 \pm 32 \%$ & $93 \pm 34 \%$ & $<0.0001$ \\
\hline Intensity of labeling in positive cases: mean \pm s.d. & $1.4 \pm 0.4$ & $2.6 \pm 1.4$ & $<0.0001$ \\
\hline \multicolumn{4}{|l|}{ Napsin A/TTF-1 joint expression ${ }^{\mathrm{a}}$} \\
\hline Napsin $\mathrm{A}(+) / \mathrm{TTF}-1(+)$ & $16(15 \%)$ & $51(85 \%)$ & $<0.0001$ \\
\hline Napsin $A(-) / T T F-1(+)$ & $47(44 \%)^{b}$ & $2(3 \%)^{\mathrm{C}}$ & \\
\hline Napsin A(+)/TTF-1(-) & 0 & 0 & \\
\hline Napsin A(-)/TTF-1(-) & $43(41 \%)$ & $7(12 \%)$ & \\
\hline
\end{tabular}

Abbreviation: s.d., standard deviation.

${ }^{\mathrm{a}} \mathrm{TTF}-1$ result was not available for six LCNECs.

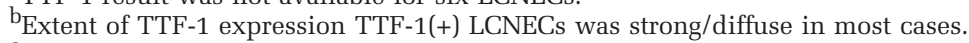

In both cases of Napsin A(-)/TTF-1(+) adenocarcinomas, TTF-1 expression was weak/focal.

consecutive sections, we confirmed in side-by-side comparison that napsin A was expressed in tumor areas that were also positive for neuroendocrine marker(s).

Clinicopathologic features of napsin A-positive LCNECs are summarized in Table 4. Patients were on average 64 years old, $65 \%$ were female, and all but one patient were smokers with a mean 44 pack-year smoking history. Following tumor resection, death of disease or tumor recurrence occurred in $53 \%$ of patients.

Genomic data were available for 14 napsin A-positive LCNECs (Table 5). Next-generation sequencing using MSK-IMPACT platform was available for nine cases (cases 9-17); the detailed molecular results for these cases have been previously reported. ${ }^{6}$ Five additional cases were analyzed by Sequenom mass spectrometry genotyping for hot-spot mutations in eight oncogenes, including KRAS. Overall, 11 of 14 tested tumors harbored mutations typical of lung adenocarcinoma-KRAS $(n=5)$, STK11 $(n=4)$ or both KRAS+STK11 $(n=2)$. One additional tumor harbored HER2 insertion-an alteration also less frequently found in lung adenocarcinomas. One additional case harbored a HER2 insertion-also alteration typical of adenocarcinoma. Conversely, $R B 1$ - the gene altered in nearly all small cell carcinomas-was intact in all but one of the cases. The sole case with $R B 1$ mutation also harbored a KRAS mutation, and had a wild-type TP53.

\section{Discussion}

In this study, we examined expression of napsin A-a marker of exocrine differentiation-in a large series of LCNECs and other lung neuroendocrine 

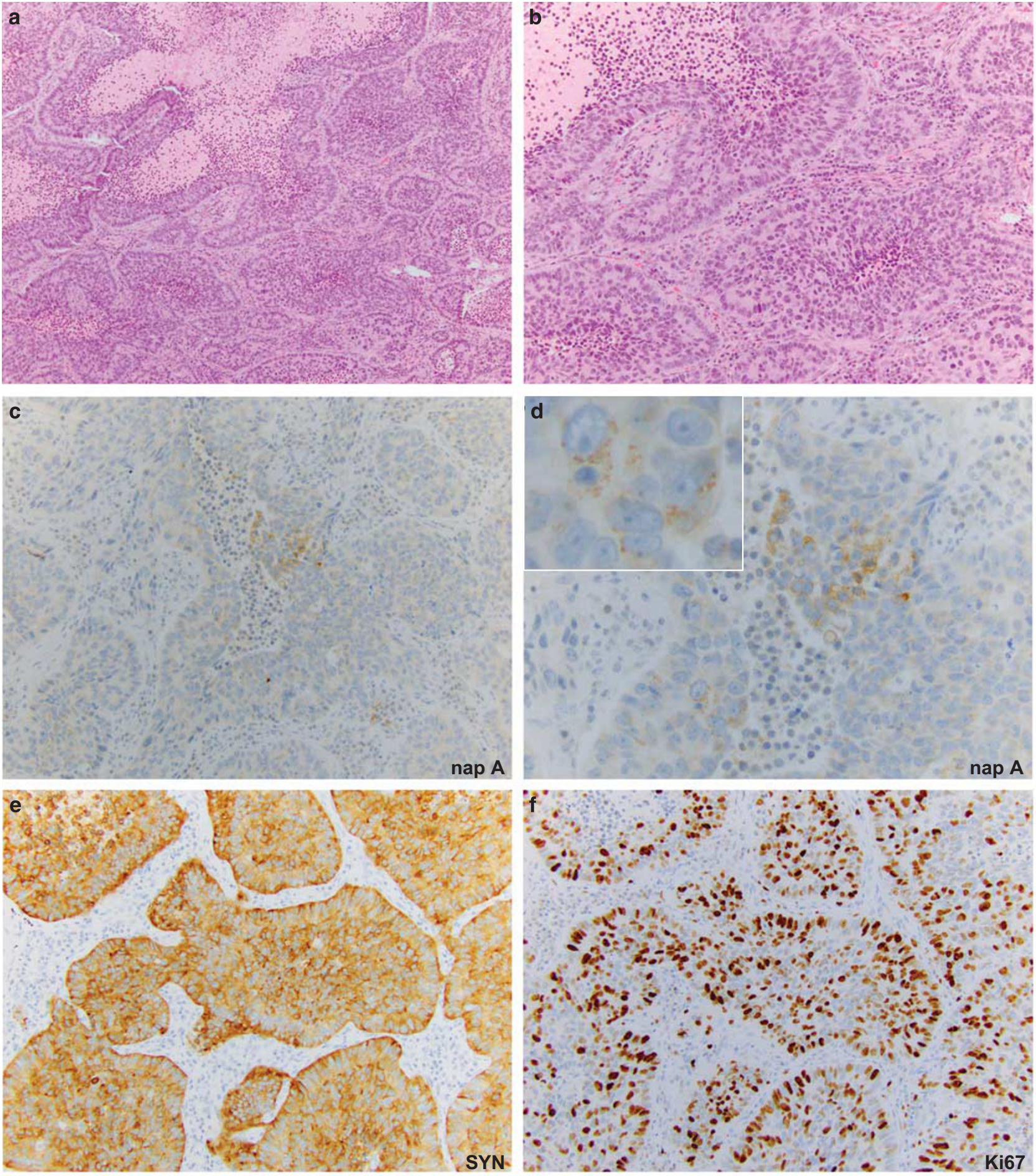

Figure 1 Example of LCNEC with focal napsin A expression (case ID 16 in Table 2). (a) H\&E sections illustrate classic LCNEC morphology, including nested growth pattern with peripheral nuclear palisading, frequent rosette-like arrangements, and areas of geographic necrosis. (b) Higher-power image illustrates non-small cell cytomorphology-moderate volume of cytoplasm and evident nucleoli. (c) and (d) illustrate weak and focal but convincing granular cytoplasmic napsin A labeling. (e) illustrates diffuse labeling for synaptophysin. (f) Ki67 marker confirms high proliferation rate $(70 \%)$.

neoplasms, and performed detailed clinicopathologic and genomic analysis of napsin A-positive cases. In agreement with prior reports, we confirmed that napsin A is consistently negative in lung carcinoids and small cell carcinomas. ${ }^{12,16}$
Conversely, we describe that while the majority of LCNECs are napsin A-negative, a subset of these tumors does express napsin A, typically at a lower level than seen in adenocarcinomas. The prevalence of napsin A expression in LCNEC was higher in 
whole-tissue sections $(21 \%)$ than in tissue microarrays $(12 \%)$, consistent with the known tendency of the latter to underestimate cases with focal reactivity. Thus, the true prevalence of napsin $\mathrm{A}$ in LCNEC is likely best reflected by the former value. We also show that the majority of napsin A-positive
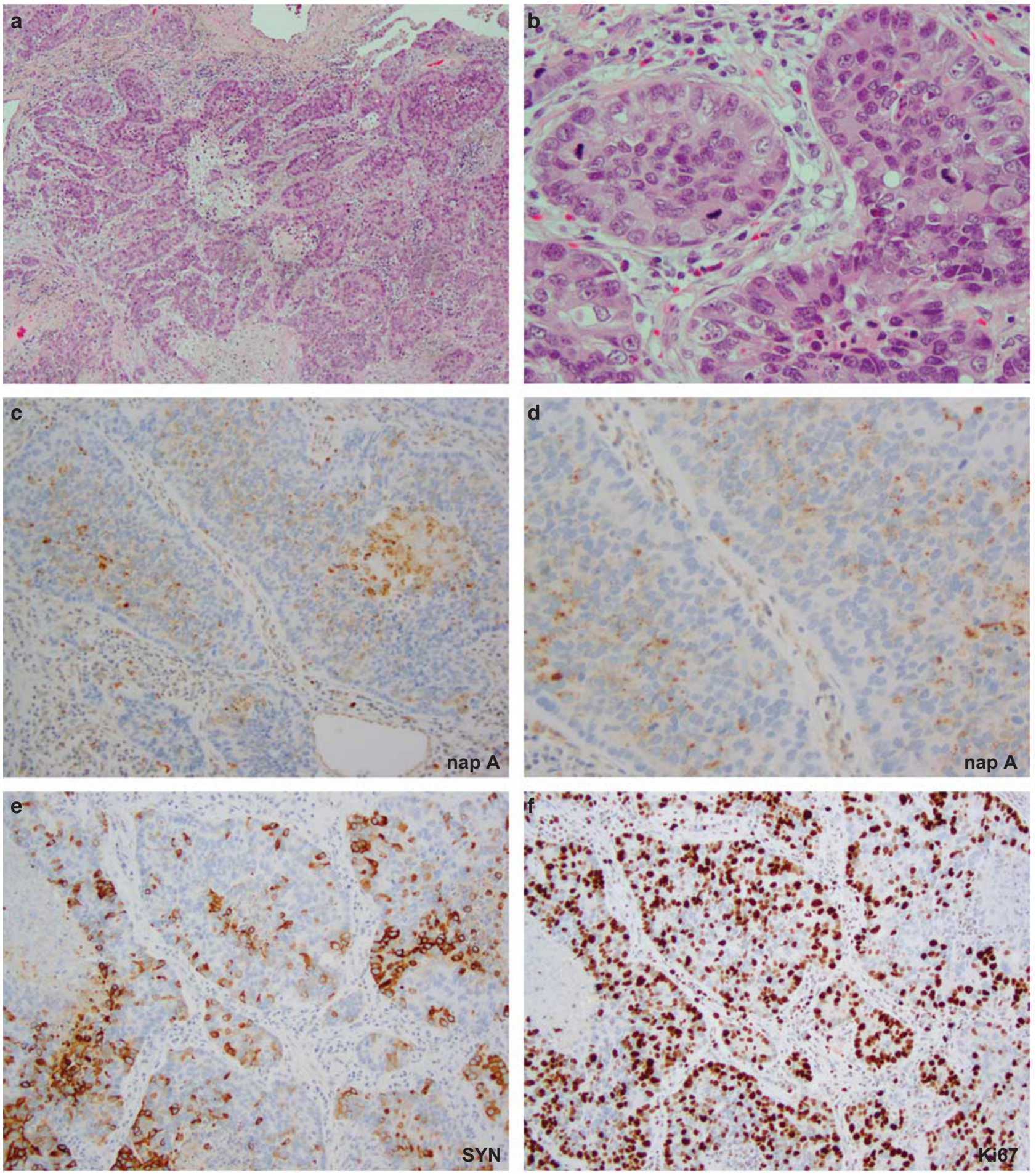

Figure 2 Example of LCNEC with diffuse weak to moderate napsin A expression (case ID 4 in Table 2). H\&E sections (a, b) illustrate neuroendocrine morphology—nested and trabecular growth pattern with peripheral nuclear palisading, and overtly non-small cell cytology_prominent nucleoli with moderate amount of cytoplasm. Tumor has amphophilic cytoplasmic commonly seen in LCNEC. This type of morphology enters in the differential diagnosis with solid adenocarcinoma. (c) and (d) illustrate napsin A labeling in the majority of tumor cells, which shows typical granular cytoplasmic reactivity with variably sized granules. Napsin A expression is seen in the absence of entrapped pneumocytes or histiocytes, confirming the specificity of labeling. (e) illustrates focal labeling for synaptophysin (SYN) in the same tumor areas as those labeling for napsin A. (f) Ki67 confirms high proliferation rate (80\%). 

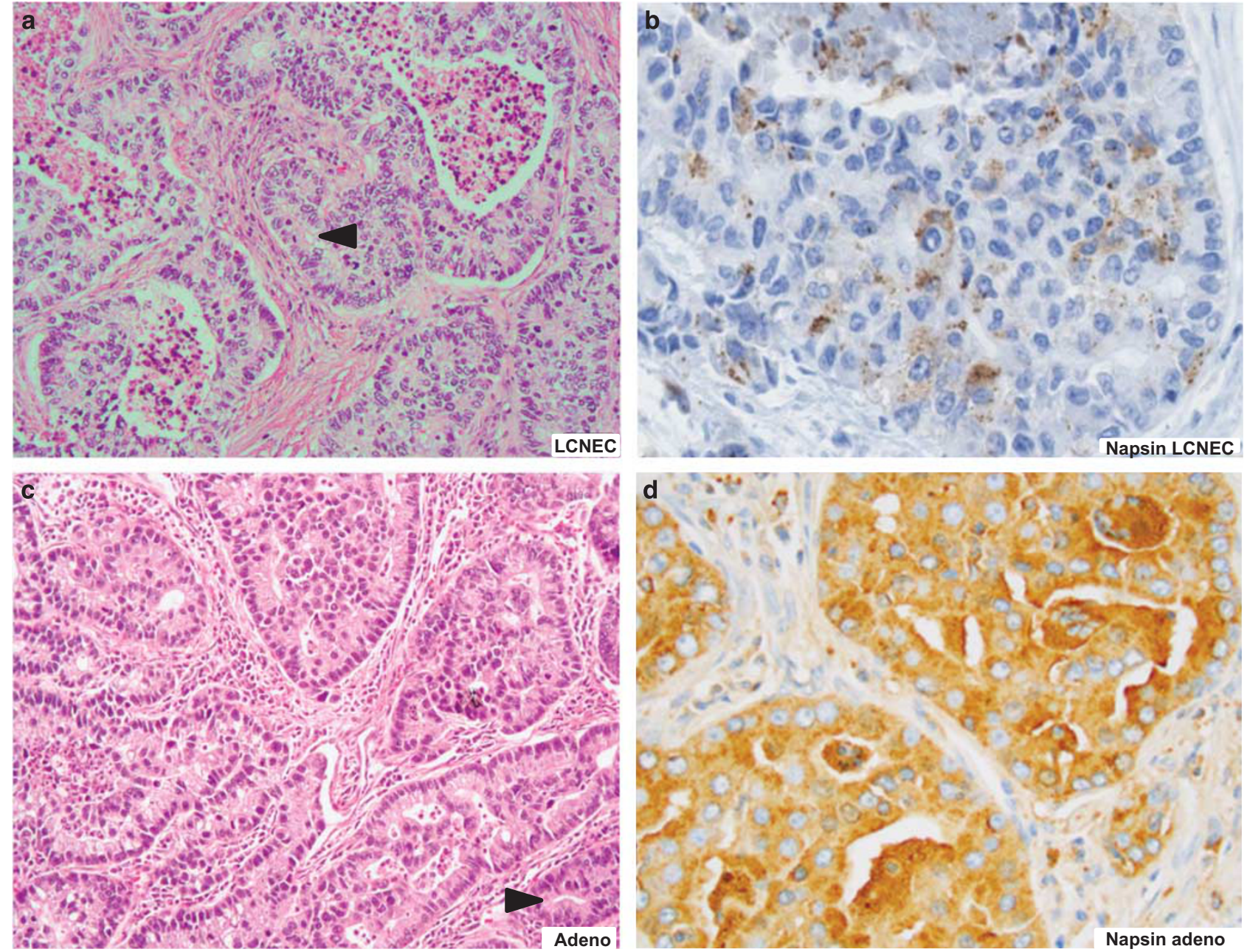

Figure 3 Contrast in intensity of napsin A reactivity in LCNEC (a and b) and lung adenocarcinoma (c and d). Intense (3+) labeling typical of adenocarcinomas (d) was not seen in any LCNECs (b). The figure illustrates a case on adenocarcinoma with cribriform pattern, which enters in the close differential diagnosis with LCNEC. Cribriform spaces in adenocarcinoma tend to have more undulating outlines, with occasional slit-like lumens, whereas luminal borders in LCNEC are characteristically rosette-like, with rigid/punched-out outlines (arrowheads). Spaces in rosettes also tend to be smaller, pinpoint-like, compared to more variable luminal sizes in adenocarcinoma.

LCNECs harbor mutations typical of lung adenocarcinoma (KRAS and/or STK11) while lacking the key alteration of small cell carcinoma (RB1).

Following the description of the criteria for LCNEC in $1991,{ }^{24}$ the biological relationship of these tumors to other major lung cancer types has been debated, ${ }^{25}$ given that they share phenotypic features with both small cell and non-small cell carcinomas. Recently, we described that molecularly, LCNEC comprises small cell-like, non-small cell/adeno-like, and in a minority of cases-carcinoid-like genomic subsets. ${ }^{6}$ These subsets were also recognized in other recent studies. ${ }^{7,26-28}$ While in prior studies, it was noted that napsin A was expressed in some LCNECs with adeno-like molecular features, ${ }^{6,7}$ here we expand on those observations in a larger series of LCNECs, focusing on clinicopathologic and genomic features of napsin A-positive cases. Overall, combined napsin A expression data and molecular findings from this and prior work ${ }^{6,7}$ support a close biological relationship and likely shared cell of origin for a subset of LCNECs and lung adenocarcinoma. Notably, in this study, we did not further address small cell-like and carcinoid-like subsets of LCNEC, and we emphasize that further studies are needed to assess the clinical utility and optimal method of identifying LCNEC subsets in routine practice. In particular, while evidence is already emerging on distinct clinical features of carcinoidlike subset, ${ }^{26}$ further work is needed to compare clinical behavior of non-small cell versus small celllike LCNEC subsets. In our prior study, we found that morphology corresponded to respective molecular subtypes in most cases, but substantial number of cases had indeterminate or mixed features. ${ }^{6}$ This emphasizes that morphologic evaluation alone-in the absence of genomic testing — can only partially predict molecular subtype of LCNEC. Nevertheless, cases with classic LCNEC morphology as defined by the WHO classification ${ }^{2}$ - organoid/trabecular 
Table 4 Clinicopathologic features of napsin A-positive large cell neuroendocrine carcinomas

\begin{tabular}{|c|c|c|c|c|c|c|}
\hline & Age & Gender & $\begin{array}{l}\text { Smoking } \\
\text { pack-years }\end{array}$ & $\begin{array}{c}\text { Tumor } \\
\text { size }(\mathrm{cm})\end{array}$ & $\begin{array}{l}p T N M \text { stage at } \\
\text { resection }\end{array}$ & Vital status \\
\hline Case 1 & 58 & $\mathrm{~F}$ & 20 & 2.5 & IIIA & NA \\
\hline Case 2 & 58 & M & 80 & 4.5 & IB & DOD (1 year survival) \\
\hline Case 3 & 66 & M & 100 & 10.2 & IIIA & DOD (1 year survival) \\
\hline Case 4 & 59 & $\mathrm{~F}$ & 39 & 5.0 & IIB & NED (8 years follow-up) \\
\hline Case 5 & 54 & M & 40 & 2.8 & IA & NED (8 years follow-up) \\
\hline Case 6 & 73 & M & NA & 5.5 & IIIA & NA \\
\hline Case 7 & 66 & $\mathrm{~F}$ & 40 & 1.6 & IIA & DOD (4 year survival) \\
\hline Case 8 & 64 & $\mathrm{~F}$ & 17 & 1.1 & IA & DOO (16 years follow-up) \\
\hline Case 9 & 73 & $\mathrm{~F}$ & 50 & 4.6 & IB & DOD (4 years survival) \\
\hline Case 10 & 51 & $\mathrm{~F}$ & 15 & 6 & IIIA & DOD (1.5 years survival) \\
\hline Case 11 & 53 & $\mathrm{~F}$ & 15 & 6.2 & IIIA & NED (6 years follow-up) \\
\hline Case 12 & 68 & M & 37.5 & 4.5 & IB & DOD (2 years survival) \\
\hline Case 13 & 67 & $\mathrm{~F}$ & 0 & 1.6 & IB & DOD (3 years survival) \\
\hline Case 14 & 67 & $\mathrm{~F}$ & 45 & 4.6 & IB & NED (4 years follow-up) \\
\hline Case 15 & 67 & $\mathrm{~F}$ & 98 & 5.2 & IB & NED (3 years follow-up) \\
\hline Case 16 & 79 & $\mathrm{~F}$ & 62.5 & 2.1 & IA & AWD (1 year follow-up) \\
\hline Case 17 & 65 & $\mathrm{M}$ & 46 & 7.8 & IIB & NED (2 years follow-up) \\
\hline Summary: & $\begin{array}{l}\text { Mean (range): } \\
64(51-79)\end{array}$ & $\begin{array}{c}\mathrm{F}: \mathrm{M} \text { ratio: } \\
11: 6\end{array}$ & $\begin{array}{l}\text { Mean (range): } \\
44(0-100)\end{array}$ & $\begin{array}{l}\text { Mean (range): } \\
4.5(1-10)\end{array}$ & $\%$ stage I: $53 \%$ & $\begin{array}{l}\% \text { disease recurrence and/or } \\
\text { death of disease: } 53 \%\end{array}$ \\
\hline
\end{tabular}

Abbreviations: AWD, alive with disease; DOD, dead of disease; DOO, dead of other causes; NA, not available; NED, no evidence of disease.

Table 5 Key genomic alterations in napsin A-positive large cell neuroendocrine carcinomas

\begin{tabular}{|c|c|c|c|}
\hline & $\begin{array}{l}\text { Alterations typical of NSCLC: } \\
\text { KRAS, STK11, other }\end{array}$ & $\begin{array}{l}\text { Alteration typical of SCLC: } \\
\text { RB1 mutation/loss }\end{array}$ & Molecular method \\
\hline Case 1 & Negative & $\mathrm{NA}^{\mathrm{a}}$ & Sequenom genotyping+Fragment analysis \\
\hline Case 2 & NA & NA & \\
\hline Case 3 & NA & NA & \\
\hline Case 4 & KRAS G12V & $\mathrm{NA}^{\mathrm{a}}$ & Sequenom genotyping+Fragment analysis \\
\hline Case 5 & ERBB2 (HER2) insertion & $\mathrm{NA}^{\mathrm{a}}$ & Sequenom genotyping+Fragment analysis \\
\hline Case 6 & NA & $\mathrm{NA}$ & \\
\hline Case 7 & KRAS G12C & $\mathrm{NA}^{\mathrm{a}}$ & Sequenom genotyping+Fragment analysis \\
\hline Case 8 & Negative & $\mathrm{NA}^{\mathrm{a}}$ & Sequenom genotyping+Fragment analysis \\
\hline Case 9 (ID 42)* & STK11 X97_splice & Absent & NGS-MSK-IMPACT \\
\hline Case 10 (ID 49)* & KRAS G12C+STK11 H168R & Absent & NGS-MSK-IMPACT \\
\hline Case 11 (ID 28)* & STK11 C278fs & Absent & NGS-MSK-IMPACT \\
\hline Case 12 (ID 32)* & KRAS G12C+STK11 V320fs & Absent & NGS-MSK-IMPACT \\
\hline Case 13 (ID 59)* & KRAS G12D & Absent & NGS-MSK-IMPACT \\
\hline Case 14 (ID 38)* & KRAS G12C & Absent & NGS-MSK-IMPACT \\
\hline Case 15 (ID 40)* & STK11 H107L & Absent & NGS-MSK-IMPACT \\
\hline Case 16 (ID 39)* & KRAS Q61L & RB1 V434fs & NGS-MSK-IMPACT \\
\hline Case 17 (ID 64)* & STK11 E165* & Absent & NGS-MSK-IMPACT \\
\hline
\end{tabular}

Abbreviations: NSCLC, non-small cell lung carcinoma; NGS, next-generation sequencing; MSK-IMPACT, Memorial Sloan Kettering-Integrated Mutation Profiling of Actionable Cancer Targets platform; NA, not available (molecular testing not performed). ${ }^{*}$ Shown in parentheses are corresponding case IDs from Rekhtman et al. ${ }^{6}$

${ }^{\mathrm{a} N A}$, Sequenom assay does not include $R B 1$ gene testing.

architecture with nuclear palisading and overtly non-small cell cytologic features (i.e. tumors with little resemblance to small cell carcinoma)-largely corresponded to non-small cell-like molecular subtype ${ }^{6}$ which is in line with morphologic features seen in tumors described in this study, as illustrated in Figures 1,2,3.

From the perspective of differential diagnosis, the key implication of our findings is for the distinction of LCNEC and adenocarcinoma. Some lung adenocarcinomas exhibit cribriform or solid growth patterns, where tumor nests may show peripheral nuclear palisading, ${ }^{29}$ thus entering in a close differential diagnosis with LCNECs (as illustrated in Figures 2 and 3). This distinction may be particularly challenging in a small biopsy specimens, where neuroendocrine architecture may be difficult to appreciate. While in prior studies, it was suggested that napsin A expression is entirely specific for adenocarcinoma and thus would exclude the diagnosis of LCNEC, ${ }^{16,18}$ our data suggest that napsin A expression, particularly if weak and focal, should not be regarded as a strict exclusion criterion for LCNEC. As such, the diagnosis should be made 
based on appropriate morphology and evidence of neuroendocrine marker expression, according to the standard criteria, ${ }^{2}$ even in the presence of low-level expression of napsin A. On the other hand, our findings do suggest that intense (3+) labeling for napsin A would indeed favor the diagnosis of adenocarcinoma since all LCNECs in this series had weak or moderate intensity of napsin A expression, and expression was focal in the majority of cases. This is in line with our observations in combined carcinomas with LCNEC and adenocarcinoma components, in which transition from adenocarcinoma to LCNEC is consistently accompanied by a sharp decline in napsin A labeling, typically manifesting as complete loss but in some cases showing partially retained expression (data not shown).

From the classification perspective, however, one may wonder whether LCNEC with napsin A expression should instead be classified as 'adenocarcinoma with neuroendocrine differentiation', since napsin A is a marker of exocrine differentiation and given the similarity in major genomic alterations between these tumors. 'Non-small cell carcinoma with neuroendocrine differentiation' is defined by WHO as conventional adeno- or squamous cell carcinoma, in which occult neuroendocrine marker expression is detected by immunohistochemistry in the absence of overt neuroendocrine morphology. This is seen in $10-20 \%$ of conventional adenocarcinomas, ${ }^{19,30,31}$ and is in line with gene expression studies consistently detecting 'neuroendocrine' subtype in non-small cell carcinomas, primarily adenocarcinomas. ${ }^{32-34}$ The major difference from that category is that cases described in this study exhibited overt neuroendocrine morphology, as described above (organoid nesting, trabeculae, rosettes, with generally monotonous nuclei despite the high proliferative rate). Another consideration supporting a separate designation for these tumors is the fact that neuroendocrine marker expression in conventional non-small cell carcinomas tends to be focal and chromogranin A expression is particularly uncommon, ${ }^{30}$ whereas almost all cases described here exhibited diffuse expression of at least one neuroendocrine marker, and most cases expressed chromogranin A at least focally. However, admittedly, some tumors meeting the criteria for LCNECs label for neuroendocrine markers only focally. In terms of clinical behavior, in this study, napsin A-positive LCNECs were associated with a high rate of post-surgical recurrence and/or death, even for stage I tumors, in line with prior data on LCNEC, ${ }^{35}$ whereas neuroendocrine marker expression in conventional non-small cell carcinomas is currently thought to bear no clear impact on prognosis. ${ }^{30}$ Lastly, even though major adeno-type alterations (KRAS, STK11) are shared between these tumors, in our prior study, we identified several molecular differences, including a particularly high rate of chromatin modifier gene alterations in adeno-like LCNECs ${ }^{6}{ }^{6}$ suggesting that while related, these tumors are also molecularly distinct. While the above considerations support a separate designation of adeno-like LCNEC and adenocarcinoma with neuroendocrine differentiation/marker expression, it is likely that these tumors represent entities in a spectrum, such that some smoking-related adenocarcinomas may undergo neuroendocrine differentiation, possibly related in part to alterations in chromatin modifier genes. As such, there may be a continuum of neuroendocrine differentiation from cases only detectable by immunohistochemistry to cases with classic LCNEC morphology, and diagnostic difficulties may indeed arise in some 'gray-zone' cases, as noted in prior publications. ${ }^{1}$ Further studies, including at the molecular level, will be needed to better define the relationship between these tumors, and to further refine the diagnostic criteria.

Another consideration from a classification perspective is whether napsin A-positive LCNEC may qualify for the diagnosis of 'combined LCNEC with adenocarcinoma.' Indeed, combined LCNEC and conventional non-small cell carcinomas, particularly adenocarcinomas, are fairly common. ${ }^{2,35}$ Against this, is the lack of morphologically recognizable conventional adenocarcinoma component in tumors described here, whereas in the current WHO classification, the diagnosis of 'combined' carcinomas is reserved for tumors with morphologically separable neuroendocrine and non-neuroendocrine components. Conceptually, however, LCNECs with napsin A expression may be analogous to the largely historic category of 'amphicrine' lung carcinomasthe term applied to tumors in which a combination of neuroendocrine and exocrine ultrastructural features is detected within a single cell by electron microscopy. ${ }^{36}$

Another helpful diagnostic observation from this study is that of a distinctive pattern of combined TTF-1/napsin A expression in LCNEC versus adenocarcinoma. While the majority of LCNECs have TTF-1-diffuse/napsin A-negative or focal profile, such profile is quite rare in adenocarcinoma, since adenocarcinomas with strong/diffuse TTF-1 expression typically also robustly express napsin A (although we have seen rare exceptions in our clinical practice, NR and PJ personal observations). Thus, this 'discrepant' expression of TTF-1-diffuse/ napsin A-negative or focal can serve as a helpful diagnostic clue to LCNEC (or small cell carcinoma), particularly in a setting of a small biopsy or cytology specimens.

Prior to this study, to our knowledge, napsin A expression had been noted only in several isolated cases of LCNEC. ${ }^{7,37,38}$ Conversely, in two prior case series that included 106 [ref. 16] and 47 [ref. 18] LCNECs, no napsin A expression was identified in any tested tumors. The difference with our study may be attributable to staining techniques and/or scoring criteria. Indeed, $>10 \%$ cutoff for percentage of positive tumor cells was used in both of those studies, and the latter study also required $2+$ or 
greater staining intensity, whereas we included cases with any extent and intensity of convincing napsin A labeling. However, different thresholds may only partially explain the difference with our study, since only 8 of 17 napsin A-positive LCNECs in our series displayed labeling in 5-10\% range, whereas other tumors displayed more diffuse labeling, albeit with weak to moderate intensity. The other potential contributing factor could be the inclusion criteria, since the diagnosis of LCNECs is known to show poor inter-observer agreement, even among experienced thoracic pathologists. ${ }^{3,5}$ We note that in three recent molecular studies, ${ }^{18,27,39}$ LCNECs harbored lower rate of KRAS mutations than seen in our series, ${ }^{6}$ further suggesting likely inter-observer variability in the current inclusion criteria for LCNEC, although regional differences in the prevalence of adeno-like subtype of LCNEC or technical differences in sequencing methods cannot be excluded.

In conclusion, here we show that LCNEC is unique among lung neuroendocrine neoplasms in that a subset of these tumors expresses napsin A, and we expand on prior finding that such tumors exhibit major genomic alterations typical of lung adenocarcinoma. Despite the apparent close biological relationship and likely shared cell of origin, adeno-like LCNECs show distinctive phenotypic properties and, based on prior data, do have some distinctive molecular alterations, supporting their classification as a separate entity from conventional adenocarcinoma. Further studies are needed to evaluate the clinical utility and method of distinguishing the subtypes of LCNEC in clinical practice.

\section{Acknowledgments}

This study was supported in part by the grant from the Fiona and Stanley Druckenmiller Center for Lung Cancer Research (to NR). This research was also made possible by infrastructure support by the NIH/ NCI Cancer Center Support Grant P30 CA008748.

\section{Disclosure/conflict of interest}

The authors declare no conflict of interest.

\section{References}

1 Rekhtman N. Neuroendocrine tumors of the lung: an update. Arch Pathol Lab Med 2010;134:1628-1638.

2 Brambilla E, Beasley MB, Chirieac LR, et al. Large cell neuroendocrine carcinoma. In: Travis WD, Brambilla E, Burke AP, et al. (eds). WHO Classification of Tumours of the Lung, Pleura, Thymus and Heart. IARC Press: Lyon, 2015, pp 69-72.

3 den Bakker MA, Willemsen S, Grunberg K, et al. Small cell carcinoma of the lung and large cell neuroendocrine carcinoma interobserver variability. Histopathology 2010;56:356-363.
4 Jiang SX, Kameya T, Shoji M, et al. Large cell neuroendocrine carcinoma of the lung: a histologic and immunohistochemical study of 22 cases. Am J Surg Pathol 1998;22:526-537.

5 Travis WD, Gal AA, Colby TV, et al. Reproducibility of neuroendocrine lung tumor classification. Hum Pathol 1998;29:272-279.

6 Rekhtman N, Pietanza MC, Hellmann MD, et al. Nextgeneration sequencing of pulmonary large cell neuroendocrine carcinoma reveals small cell carcinoma-like and non-small cell carcinoma-like subsets. Clin Cancer Res 2016;22:3618-3629.

7 Karlsson A, Brunnstrom H, Micke P, et al. Gene expression profiling of large cell lung cancer links transcriptional phenotypes to the new histological WHO2015 classification. J Thorac Oncol 2017;12: 1257-1267.

8 Brasch F, Ochs M, Kahne $\mathrm{T}$, et al. Involvement of napsin $\mathrm{A}$ in the $\mathrm{C}$ - and N-terminal processing of surfactant protein B in type-II pneumocytes of the human lung. J Biol Chem 2003;278:49006-49014.

9 Bishop JA, Sharma R, Illei PB. Napsin A and thyroid transcription factor-1 expression in carcinomas of the lung, breast, pancreas, colon, kidney, thyroid, and malignant mesothelioma. Hum Pathol 2010;41:20-25.

$10 \mathrm{Li} \mathrm{L,} \mathrm{Li} \mathrm{X,} \mathrm{Yin} \mathrm{J,} \mathrm{et} \mathrm{al.} \mathrm{The} \mathrm{high} \mathrm{diagnostic} \mathrm{accuracy} \mathrm{of}$ combined test of thyroid transcription factor 1 and Napsin A to distinguish between lung adenocarcinoma and squamous cell carcinoma: a meta-analysis. PLoS ONE 2014;9:e100837.

11 Mukhopadhyay S, Katzenstein AL. Comparison of monoclonal napsin A, polyclonal napsin A, and TTF-1 for determining lung origin in metastatic adenocarcinomas. Am J Clin Pathol 2012;138:703-711.

12 Turner BM, Cagle PT, Sainz IM, et al. Napsin A, a new marker for lung adenocarcinoma, is complementary and more sensitive and specific than thyroid transcription factor 1 in the differential diagnosis of primary pulmonary carcinoma: evaluation of 1674 cases by tissue microarray. Arch Pathol Lab Med 2012;136: 163-171.

13 Agoff SN, Lamps LW, Philip AT, et al. Thyroid transcription factor- 1 is expressed in extrapulmonary small cell carcinomas but not in other extrapulmonary neuroendocrine tumors. Mod Pathol 2000;13:238-242.

14 Folpe AL, Gown AM, Lamps LW, et al. Thyroid transcription factor-1: immunohistochemical evaluation in pulmonary neuroendocrine tumors. Mod Pathol 1999;12:5-8.

15 Kaufmann O, Dietel M. Expression of thyroid transcription factor-1 in pulmonary and extrapulmonary small cell carcinomas and other neuroendocrine carcinomas of various primary sites. Histopathology 2000;36: 415-420.

16 Masai K, Tsuta K, Kawago M, et al. Expression of squamous cell carcinoma markers and adenocarcinoma markers in primary pulmonary neuroendocrine carcinomas. Appl Immunohistochem Mol Morphol 2013;21: 292-297.

17 Zhang C, Schmidt LA, Hatanaka K, et al. Evaluation of napsin A, TTF-1, p63, p40, and CK5/6 immunohistochemical stains in pulmonary neuroendocrine tumors. Am J Clin Pathol 2014;142:320-324.

18 Rossi G, Mengoli MC, Cavazza A, et al. Large cell carcinoma of the lung: clinically oriented classification integrating immunohistochemistry and molecular biology. Virchows Arch 2014;464:61-68. 
19 Augustyn A, Borromeo M, Wang T, et al. ASCL1 is a lineage oncogene providing therapeutic targets for high-grade neuroendocrine lung cancers. Proc Natl Acad Sci USA 2014;111:14788-14793.

20 Rekhtman N, Kazi S. Nonspecific reactivity of polyclonal napsin a antibody in mucinous adenocarcinomas of various sites: a word of caution. Arch Pathol Lab Med 2015;139:434-436.

21 Rekhtman N, Tafe LJ, Chaft JE, et al. Distinct profile of driver mutations and clinical features in immunomarkerdefined subsets of pulmonary large-cell carcinoma. Mod Pathol 2013;26:511-522.

22 Cheng DT, Mitchell TN, Zehir A, et al. Memorial Sloan Kettering-integrated mutation profiling of actionable cancer targets (MSK-IMPACT): a hybridization capturebased next-generation sequencing clinical assay for solid tumor molecular oncology. J Mol Diagn 2015;17: 251-264.

23 Ordonez NG. A word of caution regarding napsin A expression in squamous cell carcinomas of the lung. Am J Surg Pathol 2012;36:396-401.

24 Travis WD, Linnoila RI, Tsokos MG, et al. Neuroendocrine tumors of the lung with proposed criteria for large-cell neuroendocrine carcinoma. An ultrastructural, immunohistochemical, and flow cytometric study of 35 cases. Am J Surg Pathol 1991;15:529-553.

25 Iyoda A, Hiroshima K, Nakatani Y, et al. Pulmonary large cell neuroendocrine carcinoma: its place in the spectrum of pulmonary carcinoma. Ann Thorac Surg 2007;84:702-707.

26 Quinn AM, Chaturvedi A, Nonaka D. High-grade neuroendocrine carcinoma of the lung with carcinoid morphology: a study of 12 cases. Am J Surg Pathol 2017;41:263-270.

27 Karlsson A, Brunnstrom H, Lindquist KE, et al. Mutational and gene fusion analyses of primary large cell and large cell neuroendocrine lung cancer. Oncotarget 2015;6:22028-22037.

28 Fernandez-Cuesta L, Peifer M, George J, et al. Genomic characterization of large-cell neuroendocrine lung tumors. World Conference on Lung Cancer 2015; abstract Oral 06.01. J Thor Oncol 2015;10:S185.
29 Moreira AL, Joubert P, Downey RJ, et al. Cribriform and fused glands are patterns of high-grade pulmonary adenocarcinoma. Hum Pathol 2014;45:213-220.

30 Ionescu DN, Treaba D, Gilks CB, et al. Nonsmall cell lung carcinoma with neuroendocrine differentiationan entity of no clinical or prognostic significance. Am J Surg Pathol 2007;31:26-32.

31 Pelosi G, Pasini F, Sonzogni A, et al. Prognostic implications of neuroendocrine differentiation and hormone production in patients with Stage I nonsmall cell lung carcinoma. Cancer 2003;97:2487-2497.

32 Bhattacharjee A, Richards WG, Staunton J, et al. Classification of human lung carcinomas by mRNA expression profiling reveals distinct adenocarcinoma subclasses. Proc Natl Acad Sci USA 2001;98:13790-13795.

33 Chen F, Zhang Y, Parra E, et al. Multiplatform-based molecular subtypes of non-small-cell lung cancer. Oncogene 2017;36:1384-1393.

34 Kosari F, Ida CM, Aubry MC, et al. ASCL1 and RET expression defines a clinically relevant subgroup of lung adenocarcinoma characterized by neuroendocrine differentiation. Oncogene 2014;33:3776-3783.

35 Sarkaria IS, Iyoda A, Roh MS, et al. Neoadjuvant and adjuvant chemotherapy in resected pulmonary large cell neuroendocrine carcinomas: a single institution experience. Ann Thorac Surg 2011;92:1180-1186.

36 Chejfec G, Capella C, Solcia E, et al. Amphicrine cells, dysplasias, and neoplasias. Cancer 1985;56:2683-2690.

37 Sakai Y, Yamasaki T, Kusakabe Y, et al. Large-cell neuroendocrine carcinoma of lung with epidermal growth factor receptor (EGFR) gene mutation and coexpression of adenocarcinoma markers: a case report and review of the literature. Multidiscip Respir Med 2013;8:47.

38 Yamada K, Maeshima AM, Tsuta K, et al. Combined high-grade neuroendocrine carcinoma of the lung: clinicopathological and immunohistochemical study of 34 surgically resected cases. Pathol Int 2014;64: 28-33.

39 Miyoshi T, Umemura S, Matsumura Y, et al. Genomic profiling of large-cell neuroendocrine carcinoma of the lung. Clin Cancer Res 2017;23:757-765. 